\title{
Infiltrative lymphocytic hypophysitis successfully treated with rituximab and mycophenolate mofetil
}

\author{
Mike Lin1, Venessa Tsang1,2,3, Janice Brewer ${ }^{4}$, Roderick Clifton-Bligh',2,3 and \\ Matti L Gild(D1,2,3
}

'Department of Endocrinology and Diabetes, Royal North Shore Hospital, New South Wales, Australia, ${ }^{2}$ Cancer Genetics Unit, Kolling Institute of Medical Research, New South Wales, Australia, ${ }^{3}$ Faculty of Health and Medicine, University of Sydney, New South Wales, Australia, and ${ }^{4}$ Department of Anatomical Pathology, Royal North Shore Hospital, New South Wales, Australia

Correspondence should be addressed to M L Gild

Email

matti.gild@sydney.edu.au

\section{Summary}

Lymphocytic hypophysitis is a rare neuroendocrine disease characterised by an autoimmune inflammatory disorder of the pituitary gland. We report a 50-year-old woman who presented with headaches and bilateral sixth cranial nerve palsies. MRI of the pituitary revealed extensive fibrosis involving the sellar and extending into both cavernous sinuses causing bilateral occlusion of the internal carotid arteries (ICA). Transphenoidal biopsy confirmed the diagnosis of infiltrative fibrotic lymphocytic hypophysitis. Symptoms resolved with high dose of oral steroids but relapsed on tapering, requiring several treatments of i.v. pulse steroids over 8 months. Rituximab combined with mycophenolate mofetil was required to achieve long-term symptom relief. Serial MRI pituitary imaging showed stabilisation of her disease without reduction in sellar mass or regression of ICA occlusion. The patient's brain remained perfused solely by her posterior circulation. This case demonstrates an unusual presentation of a rare disease and highlights a successful steroid-sparing regimen in a refractory setting.

\section{Learning points:}

- Lymphocytic hypophysitis is a rare inflammatory disorder of the pituitary gland. In exceptional cases, there is infiltration of the cavernous sinus with subsequent occlusion of the internal carotid arteries.

- First-line treatment of lymphocytic hypophysitis is high-dose glucocorticoids. Relapse after tapering or discontinuation is common and its use is limited by long-term adverse effects.

- There is a paucity of data for treatment of refractory lymphocytic hypophysitis. Goals of treatment should include improvement in symptoms, correction of hormonal insufficiencies, reduction in lesion size and prevention of recurrence.

- Steroid-sparing immunosuppressive drugs such as rituximab and mycophenolate mofetil have been successful in case reports. This therapeutic combination represents a viable alternative treatment for refractory disease.

\section{Background}

Lymphocytic hypophysitis is a rare autoimmune inflammatory disorder of the pituitary gland with an annual incidence of 1 case per 9 million. Clinical presentation of lymphocytic hypophysitis is variable and can include headache, hypopituitarism, diabetes insipidus and compression of the optic nerve or chiasm. Infiltration of the cavernous sinus can affect the third, fourth and sixth cranial nerves (1).

In rare cases, both the internal carotid artery (ICA) can become occluded with the fibrotic infiltrate. Bypass 
surgery may be required to surgically correct the occlusion (2). However, in a selected few, there is development of collateral circulation with the cerebral hemispheres supplied entirely by the vertebrobasilar arteries. These collaterals develop due to the slow progressive invasion by the inflammatory processes to both carotid arteries.

Glucocorticoids are the mainstay of therapy in lymphocytic hypophysitis but are associated with high relapse rates and long-term side effects necessitating the use of steroid-sparing agents (3). Mycophenolate mofetil and rituximab have separately proved some evidence of efficacy in case series $(4,5,6,7)$. However, we present, to our knowledge, the first case of combination rituximab and mycophenolate therapy achieving long-term clinical improvement.

\section{Case presentation}

A 50-year-old Caucasian woman was referred to our department in May 2017 for evaluation of headache and diplopia. She reported a 3-week history of persistent binocular horizontal diplopia, on a background of 12 months episodic diplopia associated with headaches. There was no visual loss or photophobia. She reported no constitutional symptoms such as fever, malaise or weight loss. Amenorrhoea had been present for 2 years, without hot flushes, insomnia or mood instability. There were no symptoms of diabetes insipidus or galactorrhoea. The patient recalled presenting to the emergency department 18 months earlier with similar symptoms. Non-contrast CT of the brain was unremarkable and she was sent home. Her symptoms resolved shortly thereafter. Past medical history was significant for migraine diagnosed 3 years ago. There is no personal or family history of autoimmune diseases.

On examination, she had a normal BMI, weighing 72 $\mathrm{kg}$ and was $170 \mathrm{~cm}$ in height. There was no evidence of Cushing syndrome or acromegaly. Upper- and lower-limb neurological examination was normal. Eye examination revealed uncorrected visual acuity of 20/15 bilaterally. Visual fields were full and pupils equal and reactive. There was no rapid afferent pupillary defect or internuclear ophthalmoplegia. Dilated fundoscopy showed preserved fundi. Ocular motility testing detected minor abduction deficits consistent with bilateral cranial nerve six palsies. All other cranial nerves were unremarkable. Our ophthalmology colleagues have described this presentation (8) and our focus here is to present the novel successful treatment of refractory lymphocytic hypophysitis.

\section{Investigation}

The patient was admitted for further evaluation. Blood test results included normal haemoglobin of $153 \mathrm{~g} / \mathrm{L}$, white cell count $5.3 \times 10^{9} / \mathrm{L}$, C-reactive protein $4.3 \mathrm{mg} / \mathrm{L}$, erythrocyte sedimentation rate was $35 \mathrm{~mm} / \mathrm{h}$ and lactate dehydrogenase $167 \mathrm{U} / \mathrm{L}$. The pituitary hormone panel (Table 1) showed hypogonadotrophic hypogonadism as well as hyperprolactinaemia in keeping with stalk pressure. There was no clinical or biochemical features of diabetes insipidus. To investigate the possibility of an underlying autoimmune disease, additional workup was undertaken. Immunoglobulin G4 (IgG4) levels were within normal range (Table 1), and anti-neutrophil cytoplasmic antibody and anti-double stranded DNA tests was negative. Secondary causes of hypophysitis such as tuberculosis, sarcoidosis or syphilis were excluded by normal Quantiferon Gold, ACE level and VDRL, respectively. We were unable to check for antipituitary antibodies (APA) in our patient due to lack of testing availability. However, APA is limited by low specificity due to its presence in pituitary tumours, idiopathic hypopituitarism and autoimmune non-pituitary diseases (9).

MRI brain reported a $30 \times 20 \mathrm{~mm}$ inflammatory infiltrative sellar and parasellar mass or mass like lesion with possible occlusion of bilateral cavernous ICAs. Further neuro-imaging with MRI angiography (MRA) of the cerebral circulation demonstrated that the parasellar mass had caused complete occlusion of both cavernous ICAs. Collateral circulation to the anterior and middle cerebral arteries was provided by posterior communicating

Table 1 Pituitary hormone profile showing hypogonatrophic hypogonadism and hyperprolactinaemia.

\begin{tabular}{|c|c|c|}
\hline & Level & Reference range \\
\hline Cortisol (morning), nmol/L & 275 & $100-535$ \\
\hline $\mathrm{ACTH}, \mathrm{ng} / \mathrm{L}$ & 22.9 & $7.2-63.3$ \\
\hline $\mathrm{TSH}, \mathrm{mIU} / \mathrm{L}$ & 0.816 & $0.4-4.0$ \\
\hline Free T4, pmol/L & 10.9 & $9.0-19.0$ \\
\hline $\mathrm{FSH}, \mathrm{IU} / \mathrm{L}$ & 5.5 & 3.0-8.1 (follicular) \\
\hline LH, IU/L & 2.3 & 1.8-11.8 (follicular) \\
\hline Oestradiol, pmol/L & $<92$ & 77-920 (follicular) \\
\hline Progesterone, $\mathrm{nmol} / \mathrm{L}$ & $<0.3$ & $<3.1$ (follicular) \\
\hline Prolactin, $\mathrm{mIU} / \mathrm{L}$ & 1640 & $110-560$ \\
\hline $\mathrm{GH}, \mu \mathrm{g} / \mathrm{L}$ & 1.9 & $<8.0$ \\
\hline IGF-1, $\mu \mathrm{g} / \mathrm{L}$ & 169 & $93-245$ \\
\hline $\operatorname{lgG} 4, \mathrm{~g} / \mathrm{L}$ & 0.63 & $0.04-0.86$ \\
\hline
\end{tabular}

IgG4 level was within reference range.

ACTH, adrenocorticotropic hormone; FSH, follicular stimulating hormone; $\mathrm{GH}$, growth hormone; IGF-1, insulin growth factor 1 ; LH, luteinising hormone; T4, thyroxine; TSH, thyroid stimulating hormone. 
arteries of the vertebrobasilar system (Fig. 1). There was no evidence of cerebral ischaemia or infarction.

These findings were in keeping with an inflammatory or infiltrative condition. Differential diagnoses for this picture include granulomatous or lymphocytic hypophysitis or IgG4 disease. No significant abnormality was found on either abdominopelvic CT or whole body PET scans. Stereotactic transphenoidal biopsy of the sellar mass was then performed and histopathology showed adenohypophysial acini separated by chronic inflammation and fibrosis (Fig. 2). There were scattered lymphocytes, plasma cells and histiocytes but no definite granulomas or multinucleate giant cells. Staining for IgG4 cells was present, but the ratio of IgG4-positive cells to total IgG cells was $19.3 \%$. This is below the cut-off for definitive diagnosis of IgG4 disease (>40\%). The presence of eight IgG-positive cells in each high power field also fell short of standard criteria ( $\geq 10$ per hpf) (10). Taken together, the histologic features and immunoprofile supported the diagnosis of lymphocytic hypophysitis.

\section{Treatment}

The patient was started on a 3-day course of i.v. pulse methylprednisolone $1 \mathrm{~g}$ daily. Her visual changes and headaches dramatically resolved. She was then discharged home on oral prednisone $50 \mathrm{mg}$ daily for 10 days with early review. Progress MRI pituitary scan at 2 weeks on therapy showed stable disease without reduction in sellar mass or regression of the bilateral cavernous ICA

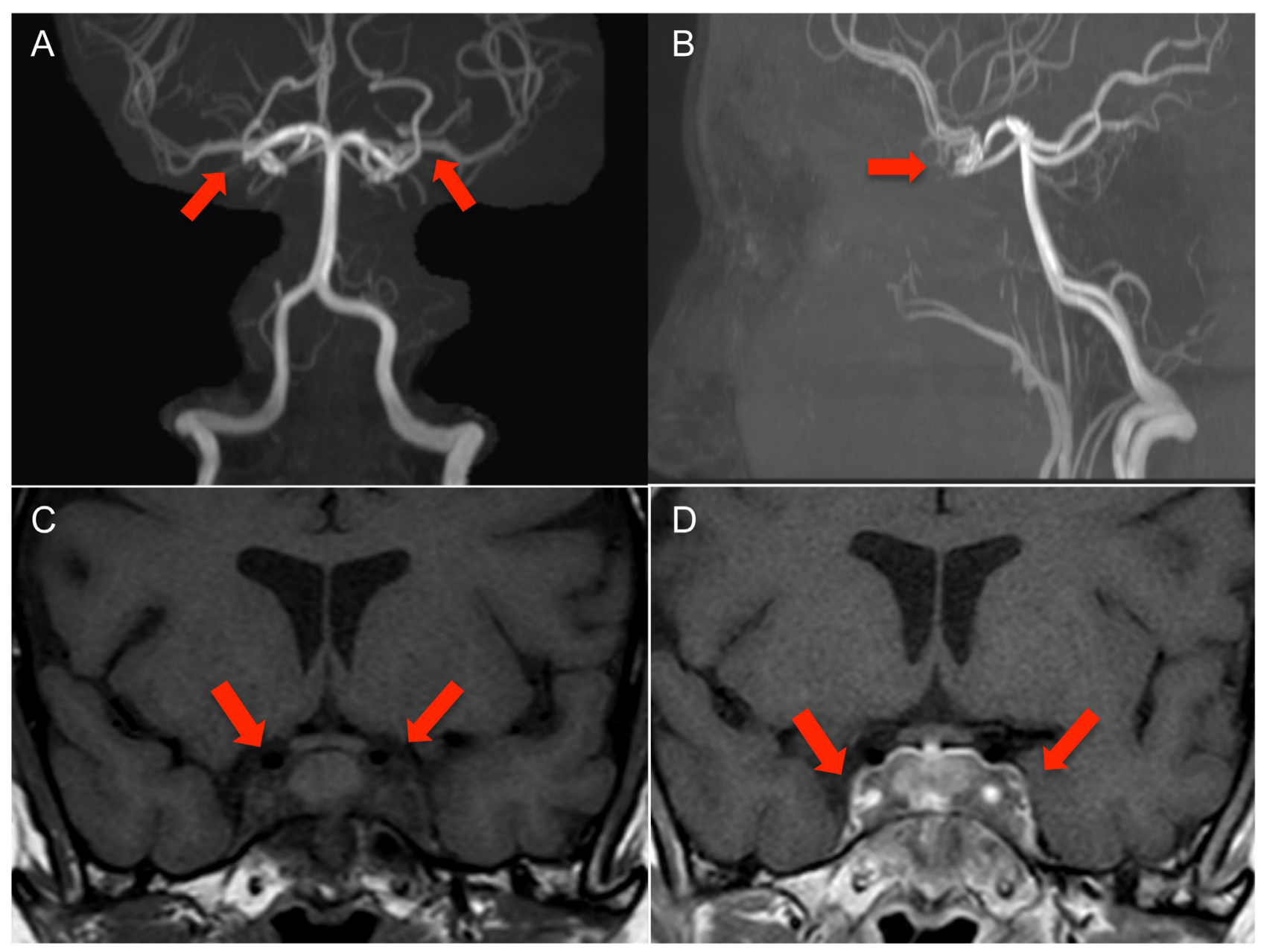

\section{Figure 1}

MR angiography with coronal (A) and sagittal (B) views showing loss of flow voids within the cavernous carotid arteries bilaterally consistent with segmental occlusion of these vessels. Circle of Willis is complete with opacification of the paired anterior cerebral and middle cerebral artery vessels via patent bilateral posterior communicating arteries. Coronal view of MRI pituitary showing infiltrative material involving the sella and extending into both cavernous sinuses. These demonstrate low signal on T1 (C) with post-gadolinium contrast enhancement (D). The signal characteristics are consistent with fibrous soft tissue. 


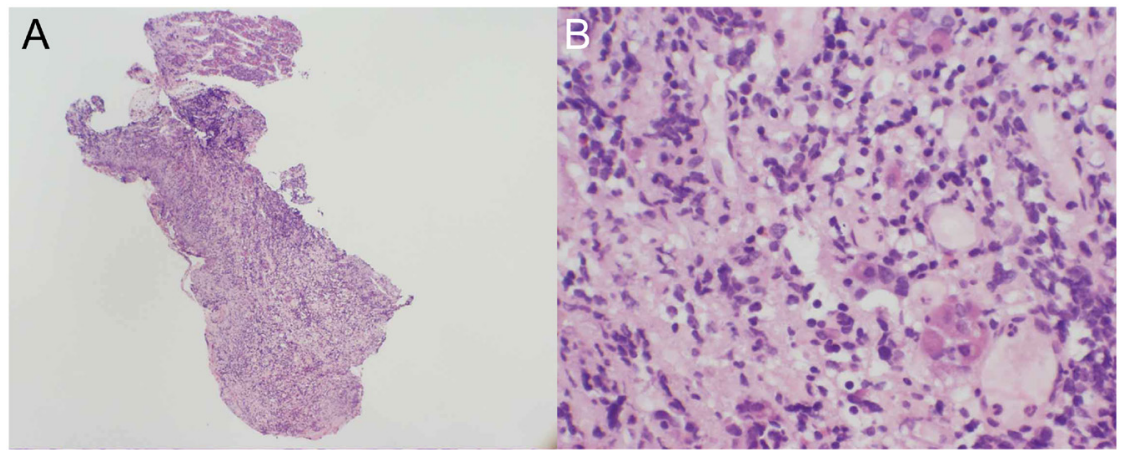

\section{Figure 2}

Haematoxylin- and eosin-stained sections of the anterior pituitary gland at $\times 40$ magnification (A) and $\times 400$ magnification (B) demonstrating scattered small nests of pituitary cells with extensive fibrosis and infiltration by numerous lymphocytes and plasma cells. occlusions. Nonetheless, given the clinical recovery, the patient was commenced on a weaning glucocorticoid regimen with the prednisone dose decreased by $12.5 \mathrm{mg}$ weekly until $15 \mathrm{mg}$ daily maintenance dose was reached.

Unfortunately, the patient reported recurrence of her severe headaches and diplopia when her steroids were tapered below $20 \mathrm{mg}$ per day, which led to two further administration of pulsed i.v. methylprednisolone treatment. MRI showed unchanged parasellar mass and persistent occluded bilateral cavernous ICAs. She also experienced significant adverse effects of glucocorticoid therapy including insomnia, Cushingoid facies and weight gain of $20 \mathrm{~kg}$. Steroid-sparing treatment was first attempted with azathioprine, but this failed to achieve long-term control of the patient's symptoms during a 5-month trial despite normal thiopurine methyltransferase (TPMT) activity and genotyping.

In May 2018 (10 months post diagnosis) our patient was commenced on weekly rituximab $100 \mathrm{mg}$ i.v. for 4 weeks based on data from published case reports $(6,7)$. She tolerated this well and was able to successfully wean prednisone down to $5 \mathrm{mg}$ daily while experiencing only infrequent mild headaches. Oral mycophenolate mofetil $100 \mathrm{mg}$ twice daily was then added as maintenance therapy. Prednisone was ceased entirely in April 2019 without evidence of secondary adrenal insufficiency. In October 2019 a single dose of i.v. rituximab $500 \mathrm{mg}$ was given on recommendation from her neuroimmunologist for the mild headaches.

\section{Outcome and follow-up}

Our patient has received interdisciplinary surveillance for the past 2.5 years. She is managed principally by the endocrinology team but has regular follow-up with immunology, neurology and ophthalmology. She remains well on maintenance MMF therapy. Her headaches have improved significantly with reduction in frequency and severity. She has no eye problems or visual changes. There have been no fevers, night sweats or infective symptoms. Her menses resumed at 12 months post diagnosis but remains irregular. After ceasing prednisone, she was able to lose $10 \mathrm{~kg}$ in weight together with improvement in her Cushingoid features. Her baseline and repeat bone mineral densities show normal $\mathrm{T}$ scores and she is on regular cholecalciferol.

Serial MRI scans of the pituitary show stable appearances of the infiltrative fibrotic changes involving the bilateral cavernous sinuses and sella. There is persistence of the bilateral ICA occlusions. The patient has been seeing a chiropractor and we have advised her not to have spinal or neck manipulation as her cerebral function is supplied solely by her posterior circulation.

The selected dose for rituximab in our patient is low in comparison to the dose used for haematological malignancies and other approved rheumatological autoimmune conditions. However, flow cytometry of B cells in our patient after rituximab treatment confirmed B cell depletion (CD19 absolute $<6 \times 10^{6} / \mathrm{L}$ ) and, therefore, immunological efficacy. Future dosing of rituximab will be based on the recurrence of symptoms or abnormalities in hormone levels. MMF will be extended for at least another 12 more months to maintain remission and possibly potentiate the effect of rituximab.

\section{Discussion}

Lymphocytic hypophysitis is a rare neuroendocrine disorder characterised by lymphocytic infiltration of the pituitary gland and chronic pituitary inflammation. The cardinal features include female predominance, onset during late pregnancy or early postpartum and association with autoimmune endocrine diseases particularly Hashimoto's thyroiditis (1). Presumptive diagnosis can be made on the basis of combined clinical, biochemical and imaging studies; however, a definitive diagnosis requires pathological examination of pituitary biopsy obtained at surgery (11). Histological features 
include plasmalymphocytic infiltration and fibrosis of the pituitary gland (1).

The symptoms of lymphocytic hypophysitis are related to the sellar mass effect. Occlusion of the internal carotid arteries is exceptionally rare and is due to the spread of the inflammatory processes into the walls of the vessels in the cavernous sinus. A literature review has identified five cases of lymphocytic hypophysitis causing ICA occlusion of which three suffered a stroke (12). To date, our patient has remained stable and retains normal cognitive and executive function. She has no complications from her ICA compression and is in remission from her neurological symptoms following treatment with glucocorticoids, azathioprine, rituximab and MMF. Given the history of similar symptoms 18 months preceding the diagnosis, it is likely that her disease was indolent, allowing arterial collaterals to develop and preventing cerebral ischaemic stroke.

Treatment with glucocorticoids remains the cornerstone of medical therapy for lymphocytic hypophysitis. Response is rapid, with recovery of pituitary function and regression of the sellar/suprasellar mass $(3,9)$. There are several limitations; however, such as disease recurrence after tapering or discontinuation of glucocorticoids occurs in up to $38 \%$ of cases (3). There are also adverse effects with long-term use including weight gain, diabetes mellitus or glaucoma. In steroid-resistant cases or when steroids cause unacceptable side effects, other immunosuppressive drugs can be considered. Azathioprine is the most frequently used agent. As a prodrug metabolised to a purine antagonist, it prevents synthesis of DNA and proliferation of lymphocytes. Most patients with lymphocytic hypophysitis respond to azathioprine $(13,14)$. One case report even showed a benefit in treating recurrent disease (14). Our patient is unusual, as azathioprine did not achieve clinical or neuroradiological improvement.

Mycophenolate mofetil (MMF) has been proposed as alternative treatment for lymphocytic hypophysitis. There have been two reported cases of its success in treating lymphocytic hypophysitis $(4,5)$. Commonly utilised in solid organ transplantation, it now plays an increasing role in treating autoimmune disorders. MMF is a prodrug of mycophenolic acid, an inhibitor of lymphocyte inosine monophosphate dehydrogenase, which restricts the proliferation of lymphocytes. Given the lymphocytic infiltrative nature of lymphocytic hypophysitis, it is expected that MMF will be effective.

The emerging role of monoclonal antibodies such as rituximab in treating lymphocytic hypophysitis is increasingly being recognised. Rituximab is an anti-CD20 MAB that causes prompt and complete depletion of peripheral B-lymphocytes. There have been two reported cases of lymphocytic hypophysitis treated successfully with rituximab $(6,7)$. In the first case, the patient had failed glucocorticoid, methotrexate and infliximab therapy. Following two courses of rituximab, there was resolution of the pituitary mass and partial recovery of the corticotrope axis. Remission was maintained during the 3 years of follow-up (6). In the second case, the patient had steroid-resistant lymphocytic hypophysitis. A course of rituximab led to recovery of visual impairment within the first few weeks and no radiological or clinical relapse of disease occurred during the next 2 years (7). Our case adds further weight to this novel treatment.

Combination of rituximab and MMF therapy has been successfully described in other autoimmune diseases such as systemic sclerosis and dermatomyositis refractory to conventional therapy $(15,16)$. To our knowledge, this is the first report, however, of this drug combination achieving long-term clinical improvement in fibrotic lymphocytic hypophysitis. We speculate that MMF is adjunctive to rituximab in increasing its efficacy and augmenting the duration of the immunosuppression. While the ICAs remained occluded in our patient, there was no evidence of neuroradiological progression. We were able to avoid the more aggressive surgical approach or stereotactic radiotherapy that is reserved for a subset of patients unresponsive to medical therapy. These treatments are associated with significant side effects such as cognitive dysfunction and iatrogenic panhypopituitarism. Finally, the paucity of available data highlights the need for further research to advance our understanding of this complex disease.

\section{Declaration of interest}

The authors declare that there is no conflict of interest that could be perceived as prejudicing the impartiality of the research reported.

\section{Funding}

This research did not receive any specific grant from any funding agency in the public, commercial or not-for-profit sector.

\section{Patient consent}

Written informed consent has been obtained from the patient for publication of the submitted article and accompanying images.

\section{Author contribution statement}

M L wrote the manuscript. M G and R C-B were the endocrinologists involved in the clinical care and contributed to the manuscript. $B \mathrm{~J}$ was the 
pathologist involved in the histopathological and immunohistochemical study of the pituitary biopsy. V T is an endocrinologist and has contributed to the manuscript.

\section{References}

1 Caturegli P, Newschaffer C, Olivi A, Pomper MG, Burger PC \& Rose NR. Autoimmune hypophysitis. Endocrine Reviews 200526 599-614. (https://doi.org/10.1210/er.2004-0011)

2 Melgar MA, Mariwalla N, Gloss DS \& Walsh JW. Recurrent lymphocytic hypophysitis and bilateral intracavernous carotid artery occlusion. An observation and review of the literature. Neurological Research 200628 177-183. (https://doi. org/10.1179/016164105X39996)

3 Honegger J, Buchfelder M, Schlaffer S, Droste M, Werner S, Strasburger C, Störmann S, Schopohl J, Kacheva S, Deutschbein T, et al. Treatment of primary hypophysitis in Germany. Journal of Clinical Endocrinology and Metabolism 2015100 3460-3469. (https:// doi.org/10.1210/jc.2015-2146)

4 Louvet C, Maqdasy S, Tekath M, Grobost V, Rieu V, Ruivard M \& Le Guenno G. Infundibuloneurohypophysitis associated with Sjögren syndrome successfully treated with mycophenolate mofetil: a case report. Medicine 201695 e3132. (https://doi.org/10.1097/ MD.0000000000003132)

5 Sawachika H, Kodama S, Mukai T \& Morita Y. Mycophenolate mofetil treatment in a patient with recurrent lymphocytic hypophysitis. BMJ Case Reports 20182018 bcr2017222678. (https://doi.org/10.1136/ bcr-2017-222678)

6 Xu C, Ricciuti A, Caturegli P, Keene CD \& Kargi AY. Autoimmune lymphocytic hypophysitis in association with autoimmune eye disease and sequential treatment with infliximab and rituximab. Pituitary 201518 441-447. (https://doi.org/10.1007/s11102-014-0592-7)

7 Schreckinger M, Francis T, Rajah G, Jagannathan J, Guthikonda M $\&$ Mittal S. Novel strategy to treat a case of recurrent lymphocytic hypophysitis using rituximab: case report. Journal of Neurosurgery 2012116 1318-1323. (doi:10.3171/2012.2.JNS111456)

8 Waldie AM, Lau O, Gild M, Little NS, Brewer J, Thieben MJ, CliftonBligh R \& Smith JEH. Lymphocytic hypophysitis presenting as bilateral abducens nerve palsy: a case report. Neuro-Ophthalmology 202044 121-124. (https://doi.org/10.1080/01658107.2018.1561721)

9 Joshi MN, Whitelaw BC \& Carroll PV. Mechanisms in endocrinology: hypophysitis: diagnosis and treatment. European Journal of Endocrinology 2018179 R151-R163. (https://doi.org/10.1530/EJE-170009)

10 Leporati P, Landek-Salgado MA, Lupi I, Chiovato L \& Caturegli P. IgG4-related hypophysitis: a new addition to the hypophysitis spectrum. Journal of Clinical Endocrinology and Metabolism 201196 1971-1980. (https://doi.org/10.1210/jc.2010-2970)

11 Howlett TA, Levy MJ \& Robertson IJ. How reliably can autoimmune hypophysitis be diagnosed without pituitary biopsy. Clinical Endocrinology 201073 18-21. (https://doi.org/10.1111/j.13652265.2009.03765.x)

12 Gendreitzig P, Honegger J \& Quinkler M. Granulomatous hypophysitis causing compression of the internal carotid arteries reversible with azathioprine and rituximab treatment. Pituitary 2020 23 103-112. (https://doi.org/10.1007/s11102-019-01005-2)

13 Lecube A, Francisco G, Rodriguez D, Ortega A, Codina A, Hernandez C \& Simo R. Lymphocytic hypophysitis successfully treated with azathioprine: first case report. Journal of Neurology, Neurosurgery, and Psychiatry 200374 1581-1583. (https://doi. org/10.1136/jnnp.74.11.1581)

14 Yang GQ, Lu ZH, Gu WJ, Du J, Guo QH, Wang XL, Ba JM, Dou JT, Mu YM \& Lu JM. Recurrent autoimmune hypophysitis successfully treated with glucocorticoids plus azathioprine: a report of three cases. Endocrine Journal 201158 675-683. (https://doi.org/10.1507/ endocrj.k10e-334)

15 Fraticelli P, Fischetti C, Salaffi F, Carotti M, Mattioli M, Pomponio G \& Gabrielli A. Combination therapy with rituximab and mycophenolate mofetil in systemic sclerosis. A single-centre case series study. Clinical and Experimental Rheumatology 201836 (Supplement 113) 142-145. (https://doi.org/10.1136/annrheumdis2016-eular-4642)

16 Parziale N, Kovacs SC, Thomas CB \& Srinivasan J. Rituximab and mycophenolate combination therapy in refractory dermatomyositis with multiple autoimmune disorders. Journal of Clinical Neuromuscular Disease 201113 63-67. (https://doi.org/10.1097/ CND.0b013e318221259d)

Received in final form 20 March 2020 Accepted 29 May 2020 